ArtefaCToS. Revista de estudios de la ciencia y la tecnología

eISSN: $1989-3612$

Vol. 7, No. 2 (2018), 2a Época, 83-105

DOI: http://dx.doi.org/10.14201/art20187283105

\title{
Los problemas de la inducción pesimista. Verdad y certeza en el debate entre el realismo y el anti-realismo
}

\author{
What is Wrong with the Pessimistic Induction? Truth and \\ Certainty in the Realism versus Antirealism Debate
}

\section{Sergio URUEÑA LÓPEZ}

Universidad del País Vasco UPV/EHU

sergio.uruena@ehu.eus

Recibido: 16/07/2018. Revisado: 17/09/2018. Aceptado: 09/10/2018

\section{Resumen}

Este trabajo presenta un análisis del debate entre realistas y anti-realistas tomando como objeto específico de estudio la inducción pesimista y el argumento del no-milagro. La hipótesis principal que se defenderá es que la aparente falta de consenso entre realistas y anti-realistas a la hora de abordar el problema de la veracidad de nuestras teorías científicas en el marco de este debate se debe en parte al uso de diferentes marcos conceptuales en torno a la verdad. Concretamente, se argumentará que la inducción pesimista es un argumento escéptico construido sobre un concepto de verdad basado en la certeza que resulta inoperativo para entender la práctica científica. El trabajo se estructura de la siguiente manera: en primer lugar se caracteriza la inducción pesimista y el argumento del no-milagro; a continuación se presentan las críticas más significativas realizadas a ambos argumentos, así como las réplicas planteadas a los mismos; finalmente, se analiza y se valora el concepto de verdad subyacente a cada uno de ellos.

Palabras clave: argumento del no-milagro; meta-inducción pesimista; meta-análisis; epistemología; realismo científico.

\begin{abstract}
This paper presents an analysis of the debate between realists and anti-realists taking as specific object of study the pessimistic induction and the no-miracles argument. The hypothesis of the paper is that the apparent lack of consensus between realists and anti-realists in addres-
\end{abstract}




$$
\begin{gathered}
\text { Sergio Urueña López } \\
\text { Los problemas de la inducción pesimista. Verdad y certeza en el debate } \\
\text { entre el realismo y el anti-realismo }
\end{gathered}
$$

sing the problem of the veracity of our scientific theories is to some extent related to the use of different conceptual frameworks on the truth. Specifically, I will argue that Laudan's pessimistic induction is a skeptical argument built on a certainty-based concept of truth which is inoperative for understanding the scientific practice. The paper is structured as follows: first, the pessimistic induction and the no-miracles argument are characterized, then the most significant criticisms of both arguments and the replies will be presented and, finally, the validity of the concept of truth underlying those arguments will be briefly analyzed and evaluated.

Keywords: no-miracles argument; pessimistic meta-induction; meta-analysis; epistemology; scientific realism.

\section{Introducción}

El debate entre el heterogéneo grupo de posiciones filosóficas que suelen catalogarse dentro de la dicotomía realismo / anti-realismo científico tiene hondas raíces históricas. Ríos de tinta han corrido desde sus inicios, especialmente en lo concerniente tanto a (i) la determinación de los compromisos que definen o deberían de definir a ambas posturas filosóficas, como a (ii) la elucidación de su legitimidad y robustez. La diversidad de posiciones y argumentos posibles en torno a (i) y (ii) no sólo ha promovido que el debate siga manteniéndose vivo en el ámbito académico, sino que este muestre una gran fertilidad filosófica tanto en términos intensionales como extensionales. En contra del pronóstico realizado en los años 80 por Arthur Fine (1984), el realismo científico y el anti-realismo siguen siendo posturas vivas, y los argumentos y réplicas propuestos que tratan de estimular el debate han aumentado considerablemente.

Respecto a la problemática existente en torno a (i) la determinación de los compromisos que caracterizan (o deberían de caracterizar) a las diferentes posturas realistas y anti-realistas, podría expresarse de manera simplificada que, a pesar de que existen distintas variedades de realismos y anti-realismos que emergen como resultado de la combinación de los posibles matices posicionales a los que cabría adherirse en el plano epistemológico, ontológico y semántico (véase González, 1993; Diéguez, 1998a, 145-156), todos ellos comparten una serie de notas comunes mínimas. Mientras que las posturas realistas tienden a considerar que la mejor manera de interpretar nuestras teorías científicas es suponiendo que estas nos proporcionan cierto acceso cognitivo a la realidad (en minúsculas), las posturas anti-realistas comparten la nota común de negar o poner en duda la solidez de esta tesis mínima con la que se compromete el realismo. 


$$
\begin{gathered}
\text { Sergio Urueña López } \\
\text { Los problemas de la inducción pesimista. Verdad y certeza en el debate } \\
\text { entre el realismo y el anti-realismo }
\end{gathered}
$$

La literatura tampoco muestra cierto consenso a la hora de (ii) elucidar la legitimidad y robustez de estas posturas filosóficas. La variedad de argumentos ofrecidos ya sean para respaldarlas o para criticarlas es sumamente amplio (véase Chakravartty, 2017). No obstante, hay dos que suelen ser reconocidos como los más poderosos: el argumento del no-milagro ("ANM" a partir de ahora), a favor del realismo, y el argumento de la inducción pesimista ("IP" a partir de ahora), en su contra (Dicken, 2016; Psillos, 1996, S306). Si bien ambos suelen presentarse como argumentos singulares y bien caracterizados, es conveniente señalar que en la literatura especializada existen diferentes formas de comprender ANM (véase Psillos, 1999, cap. 4), así como al menos cuatro versiones de IP (véase Wray, 2015), lo que complica aún más si cabe el debate.

Esta falta de consenso en torno a la caracterización de IP/ANM afecta de un modo directo tanto a la determinación del alcance de los argumentos (a qué ámbito de la ciencia en particular afectan y con qué grado) como a la justificación de su respectiva validez. Pronunciarse sobre qué premisas constituyen IP/ANM es un acto de posicionamiento que constriñe ex ante el debate a una muestra específica del universo de tipos de "IPs" y "ANMs" existentes. En este sentido, cualquier juicio sobre estos argumentos debe de comprenderse y valorarse dentro de su propia tipología específica, pudiendo requerir para cada una de sus caracterizaciones existentes fórmulas justificativas (y contra-argumentativas) diferenciadas.

El presente trabajo tiene como principal objetivo realizar un (meta)análisis del debate entre el realismo y anti-realismo tomando como objeto de estudio específico la pugna existente entre la IP clásica esgrimida por Laudan en $A$ Confutation of Convergent Realism (1981) y el ANM ideado por Putnam. Grosso modo, mientras que ANM pretende justificar la consideración de la veracidad de nuestras teorías científicas actuales como la mejor explicación de su éxito empírico (Maxwell, 1962, 18; Smart, 1963, 39; Putnam, 1975, 73), IP trata de deslegitimar la conexión "éxito empírico-verdad" que el realista supone en ANM teniendo en cuenta el hecho de que la gran mayoría de las teorías científicas actualmente consideradas falsas fueron en su momento histórico, y del mismo modo que son las actuales, aceptadas por ser empíricamente exitosas (Poincaré, [1905] 2001, Putnam, 1976, 184; Laudan, 1977, 126; 1981). La hipótesis central que se defenderá es que el realismo puede asumir IP fácilmente siempre y cuando opere con un concepto de verdad distinto del que sustenta a este argumento (esto es, que la IP de Laudan es efectiva sí y sólo sí el realismo asume el mismo concepto de verdad sobre el que este argumento se construye). En concreto, se defenderá que mientras IP es un argumento de corte escéptico construido sobre un concepto de verdad que encuentra su elemento de (des)legitimación epistémica básico en la certeza, ANM - en concordancia con Levin (1984)- no se encuentra necesariamente 


$$
\begin{aligned}
& \text { Sergio Urueña López } \\
& \text { Los problemas de la inducción pesimista. Verdad y certeza en el debate } \\
& \text { entre el realismo y el anti-realismo }
\end{aligned}
$$

vinculado a ningún concepto de verdad, lo que otorga una mayor flexibilidad interpretativa, pudiendo continuar siendo un argumento válido para defender la razonabilidad de un realismo científico prudente y crítico.

A la hora de tratar de fundamentar la hipótesis anteriormente mencionada se procederá del siguiente modo. En primer lugar, se mencionarán las diferentes dimensiones que cabría destacar de ambos argumentos, centrándonos específicamente en la caracterización de los mismos (sección 2). Seguidamente, se explorará el debate existente en torno a la justificación de IP/ANM, analizando algunas de las principales réplicas y contra-réplicas más significativas que se les ha planteado a ambos argumentos (sección 3). En tercer lugar, se analizará el concepto de verdad subyacente a IP, y se evaluarán sus insuficiencias para interpretar la actividad científica y combatir ANM (sección 4). Finalmente, se enumerarán una serie de conclusiones principales que podrían extraerse de este trabajo (sección 5).

Se espera con todo ello "arrojar algo de luz" sobre el debate entre el realismo y el anti-realismo en lo que respecta a la IP laudaniana y el ANM y, de manera más específica, explicitar cómo uno de los grandes debates de la Historia de la Filosofía de la Ciencia podría estar condicionado en su desarrollo por el compromiso con diferentes concepciones de la "verdad". Las limitaciones de este trabajo son claras: no se pretende aquí desarrollar un concepto de verdad alternativo; tampoco identificar el conjunto de "elementos" que podrían dotar a la actividad científica de robustez epistémica. Si bien estas tareas son y serán de gran importancia para el programa realista, rebasarían los límites (formales y temporales) de este trabajo.

\section{La caracterización de la inducción pesimista y del argumento del no-milagro}

Desde su surgimiento, ANM e IP han propiciado la emergencia de una gran diversidad de críticas y refinamientos que podrían ser agrupados según su objetivo central en, al menos, tres planos teóricos: (i) la caracterización, (ii) el alcance y (iii) la justificación. Respectivamente, el objetivo de cada dimensión es: (i) determinar cuáles son los propósitos, premisas, conclusiones y forma lógica del argumento en cuestión, (ii) identificar cuál es su rango de aplicabilidad, y (iii) evaluar su robustez.

Tal y como se apuntó en la introducción, la caracterización es una de las dimensiones más importantes del debate, pues este implica fijar el marco general en el que cabrían que se manifiestasen el resto de dimensiones. Se pueden utilizar diferentes argumentos a la hora de fundamentar la misma posición, $\mathrm{y}$ esos distintos argumentos, a su vez, pueden poseer diversos grados de validez que podrían requerir herramientas analíticas o contra-argumentativas individualizadas para cada uno de ellos. Así, caracterizar de un modo correcto un 


$$
\begin{gathered}
\text { Sergio Urueña López } \\
\text { Los problemas de la inducción pesimista. Verdad y certeza en el debate } \\
\text { entre el realismo y el anti-realismo }
\end{gathered}
$$

argumento rival merece gran atención, entre otras cosas, para evitar cometer la falacia del hombre de paja y que las críticas planteadas sean realmente efectivas.

¿Cómo debemos, pues, caracterizar ANM e IP? Tal y como se explicitó anteriormente, aquí nos centraremos de manera específica en IP y ANM clásicos: la ideada por Larry Laudan (1981) y Hilary Putnam (1976) respectivamente. A la hora de caracterizarlos, se acudirá a las fuentes originales y extraeremos de ellas mismas la estructura básica del argumento. La cuestión de la robustez justificativa será posteriormente discutida en la tercera sección.

\subsection{El argumento del no-milagro}

El argumento del no-milagro, también reconocido en la literatura especializada como "el último argumento" o "el argumento del milagro", encuentra sus antecedentes más inmediatos en John Smart $(1963,39)$, para quien explicar el éxito de las teorías científicas sin apelar a criterios de verdad implicaría creer en una especie de "coincidencia cósmica": ¿Cómo es posible que las teorías científicas sean tan exitosas (en términos predictivos y explicativos) si estas no son verdaderas? Siguiendo esta misma lógica, Hilary Putnam estimó, cuando aún podía ser considerado un aliado del realismo, que a la hora de interpretar la actividad científica esta era "la única filosofía que no hace del éxito de la ciencia un milagro" (Putnam, 1975, 73; énfasis añadido).

La idea básica que subyace a ambas propuestas es que a la hora de explicar la actividad científica el realismo se posiciona como la hipótesis interpretativa más plausible o razonable. Años más tarde, Richard Boyd (1984) recogería esta misma idea y reformularía el argumento de Putnam siguiendo el esquema lógico propio de lo que se ha denominado como Inferencia a la Mejor Explicación ("IME" a partir de ahora) ${ }^{1}$. No obstante, tal y como señala Carman (2006, 94; 2016), cabe matizar a este respecto que la propuesta de Boyd difiere parcialmente de la de Putnam. En concreto, para Putnam lo que sólo puede ser explicado desde el realismo es el sucesivo éxito empírico que tienen las teorías científicas, mientras que para Boyd no es tanto la verdad de manera directa lo que mejor explica el realismo, sino más bien el valor instrumental de la metodología científica (véase Boyd,1984, 59).

\footnotetext{
${ }^{1}$ No obstante, tal y como sugiere Paula Olmos, bautizar este tipo de argumentos bajo la expresión "inferencia a la mejor explicación" no resulta una acción del todo adecuada. Entre diversas razones, ello se debe a que las inferencias hacen referencia a procesos psicológicos individuales (solipsistas), y los actos de argumentación son ante todo actos comunicativos que se dan en la esfera pública y que poseen, por lo tanto, un claro carácter dialógico (Olmos, pendiente de publicación).
} 


$$
\begin{gathered}
\text { Sergio Urueña López } \\
\text { Los problemas de la inducción pesimista. Verdad y certeza en el debate } \\
\text { entre el realismo y el anti-realismo }
\end{gathered}
$$

En cualquier caso, interpretar el argumento del no-milagro putnamiano como un argumento que comparte la forma básica de una IME puede ser de gran ayuda a la hora de caracterizar el argumento de manera más precisa. Tal y como ya ha apuntado Stathis Psillos (1999, cap. 4), especificar el ANM no es una tarea sencilla debido a la pluralidad de matices y diferentes formulaciones del argumento que se promueven y se han promovido a lo largo de la historia (por ejemplo: Brown, 1982; Musgrave, 1988; Lyons y Clarke, 2002, xii; Lyons, 2003; Worrall, 2005; Dellsén, 2016). Pero a pesar de esta diversidad que forma parte natural del desarrollo de la práctica y estrategia argumentativa misma, Psillos también destaca que existen diversos puntos comunes entre las diversas formulaciones del argumento:

Independientemente de la forma exacta mediante la que se presente el argumento, la idea central es que el éxito de las teorías científicas sirve como soporte a las dos tesis siguientes: (a) las teorías científicas deben de interpretarse de manera realista y, (b) así interpretadas, estas teorías son aproximadamente verdaderas. (Psillos, 2009, 48; traducción propia)

No obstante, conviene tener en cuenta las principales formas concretas que estas bases comunes ocultan, especialmente a través de (b). Si bien cabría considerar que cada una de las formalizaciones de ANM son distintos modos de fundamentar la razonabilidad de considerar nuestras teorías científicas como verdaderas, el modo de comprender esta verdad ha sido variable. Esta variabilidad viene dada a la hora de interpretar la fuerza de la conclusión del argumento.

De manera general, podríamos considerar que ANM encaja en el esquema pierceano de una IME (Pierce, 1958, 189): se comienza con una observación o hecho que pretende ser explicado (E), se postula una o varias hipótesis explicativas no trivialmente rivales $\left(\mathrm{H} ;=\left\{\mathrm{H}_{1}, \ldots, \mathrm{H}_{\mathrm{n}}\right\}\right)$ y, a partir de ahí y en base a una batería de presupuestos y condicionantes contextuales que participan en la práctica argumentativa, debatimos en torno a cuál de la serie de hipótesis 'H;' posee mejores virtudes explicativas para 'E'. Una vez que el peso de las evidencias y/o de los argumentos presentados muestren qué hipótesis resulta más explicativa/predictiva/etc. (p. ej.: ' $\mathrm{H}_{1}$ '), postulamos de manera tentativa que tenemos motivos suficientes para sostener en ese contexto (y si las condiciones no cambian) que ' $\mathrm{H}_{1}$ '.

En el caso del argumento del no-milagro, el anterior esquema funciona del siguiente modo. Nos encontramos con la evidencia del éxito regular de la ciencia (E), la cual pretendemos explicar desde la Filosofía de la Ciencia. Para ello, contamos con una serie de hipótesis candidatas que podemos englobar dentro de los paraguas generales de "realismo científico" $\left(\mathrm{H}_{\mathrm{R}}\right)$ y "anti-realismo" $\left(\mathrm{H}_{\mathrm{A}}\right)$. A partir de ahí, y en base a que el realismo es la postura 


$$
\begin{aligned}
& \text { Sergio Urueña López } \\
& \text { Los problemas de la inducción pesimista. Verdad y certeza en el debate } \\
& \text { entre el realismo y el anti-realismo }
\end{aligned}
$$

"que no hace del éxito de la ciencia un milagro" (Putnam, 1975, 73) podemos considerar de manera tentativa (a) que "las teorías científicas deben ser interpretadas de manera realista y (b) que así interpretadas, estas teorías son aproximadamente verdaderas" (Psillos, 2009, 48). Pero, tal y como decíamos, (b) puede interpretarse de diferentes maneras o bajo diferentes gradientes de radicalidad. Por ejemplo, puede interpretarse (b1) que las teorías son de hecho verdaderas $\left(\mathrm{H}_{\mathrm{R}}\right)$, (b2) que son probablemente verdaderas $\left[\operatorname{Pr}\left(\mathrm{H}_{\mathrm{R}} \mid \mathrm{E}\right)\right.$ $>\operatorname{Pr}(\mathrm{H} ; \mid \mathrm{E})]$ o (b3) que resulta plausible o razonable, por ahora, considerarlas como verdaderas $\left(\sim \mathrm{H}_{\mathrm{R}}\right)$.

\subsection{La inducción pesimista}

El argumento de la inducción pesimista, también denominado en la literatura especializada como "meta-modus tollens pesimista", "meta-inducción pesimista", o "inducción histórica pesimista" ha sido considerado por varios autores como uno de los argumentos más fuertes en contra del realismo científico (Worrall, 1989, 99; Psillos, 1996, S306; Kitcher, 1993, 136; Leplin, 1997, 136; Lange, 2002, 281; Magnus y Callender, 2004, 322). No obstante, y al contrario de lo que sucedía en el caso de ANM, no existe un elemento común suficientemente robusto (más allá de basarse todas ellas en la Historia de la Ciencia) que permita unificar los diferentes argumentos considerados bajo el rótulo de "inducción pesimista". En concreto, Wray (2015) ha identificado hasta cuatro versiones del argumento.

(I) La primera versión de IP, atribuida a a Larry Laudan (1981) -aún cuando Laudan no la haya planteado de manera explícita (Wray, 2015, 65)- suele ser típicamente caracterizada como reductio ad absurdum de la tesis realista. Esto es, IP partiría asumiendo la tesis realista de que el éxito empírico de una teoría es una prueba de su verdad para después negarla aportando evidencia empírica sobre casos de la Historia de la Ciencia en los que teorías hoy consideradas falsas fueron consideradas exitosas en el pasado (véase Psillos, 1999, 102). No obstante, esta versión puede reconstruirse a partir de un análisis de A Confutation of Convergent Realism también como un argumento deductivo (véase Lyons, 2002): IP partiría del hecho de que han existido teorías científicas (t) que, aun siendo falsas $(\neg \mathrm{V})$, resultaron ser empíricamente exitosas (S) para concluir que no toda teoría exitosa puede ser considerada verdadera.

$$
\frac{\exists \mathrm{t}(\mathrm{St} \wedge \neg \mathrm{Vt})}{\therefore \neg \forall \mathrm{t}(\mathrm{St} \rightarrow \mathrm{Vt})}
$$

(II) La segunda de las versiones de IP correspondería con la presentada por Putnam (1976, 181-185). Esta forma de IP plantea la posibilidad de que la ratio de falsedad de las teorías pasadas pueda trasladarse a las actuales: 


$$
\begin{aligned}
& \text { Sergio Urueña López } \\
& \text { Los problemas de la inducción pesimista. Verdad y certeza en el debate } \\
& \text { entre el realismo y el anti-realismo }
\end{aligned}
$$

¿Qué ocurriría si todas las entidades teóricas postuladas por una generación (moléculas, genes, etc., así como electrones) resultan 'no existir' desde el punto de vista de una ciencia posterior? (...) Una razón por la que esto es una preocupación seria es que con el tiempo la siguiente meta-inducción se vuelve abrumadoramente convincente: al igual que ningún término que se utilizaba en la ciencia hace más de 50 años (o los que sean) refería, resultará que ningún término que es utilizado ahora (exceptuando los términos observacionales, si es que los hay) refiere (Putnam, 1976, 183-184; traducción propia).

(III) La tercera IP fue presentada por Henry Poincaré ([1905] 2001), y su objetivo no es atacar al realismo, sino señalar un hecho histórico sobre la ciencia:

La naturaleza efímera de las teorías científicas toma por sorpresa al hombre mundano. Cuando termina el breve periodo de prosperidad, ve cómo una por una las teorías son abandonadas; ve ruinas amontonadas sobre ruinas; predice que las teorías actuales también sucumbirán en poco tiempo, y concluye que son absolutamente en vano. Esto es lo que llama la bancarrota de la ciencia. (Poincaré, [1905] 2001, 122; énfasis en el original; traducción propia).

(IV) Finalmente, podría considerarse como una cuarta $1 \mathrm{P}$ el argumento de las alternativas no concebidas presentado por Standford (2001; 2006):

Propongo la siguiente Nueva Inducción sobre la Historia de la Ciencia: que a lo largo de la historia de la investigación científica, y en prácticamente todos los campos, hemos ocupado repetidamente una posición epistémica en la que pudimos concebir sólo una o unas pocas teorías que estaban bien confirmadas por las pruebas disponibles, mientras que la historia de la investigación ha revelado sistemáticamente (si no invariablemente) que existían otras alternativas radicalmente distintas a aquellas seleccionadas que hubieran estado tan bien confirmadas por las evidencias previamente disponibles como las teorías que nos inclinábamos a aceptar sobre la base de esas mismas pruebas. (Stanford, 2001, S9; traducción propia).

Esta cuarta IP nos invita a pensar que "tenemos todas las razones para creer y ninguna razón para dudar que probablemente esta es también nuestra situación actual" (Saatsi et al., 2009, 379-380): las teorías actuales que consideramos verdaderas no son más que una elección entre otras muchas alternativas existentes no concebidas que logran del mismo modo "salvar los fenómenos". 


$$
\begin{aligned}
& \text { Sergio Urueña López } \\
& \text { Los problemas de la inducción pesimista. Verdad y certeza en el debate } \\
& \text { entre el realismo y el anti-realismo }
\end{aligned}
$$

Tal y como se anticipó en la introducción, aquí nos centraremos específicamente en (I), la meta-inducción pesimista laudaniana.

\section{EI debate en torno a la justificación de IP/ANM}

A la hora de evaluar la robustez de IP y ANM, las estrategias más populares utilizadas por los autores de referencia han sido dos: o bien mostrar la disconformidad hacia alguna o algunas de las premisas que sustentan el argumento del adversario, o bien exponer que de ellos no se sigue la conclusión pretendida (esto es, mostrar que el argumento no es lógicamente válido).

Si bien el objetivo del presente trabajo es identificar el concepto de verdad subyacente a ambos argumentos para en primer lugar analizarlos y después evaluarlos, ello se conseguirá una vez mostremos las principales críticas y réplicas ofrecidas para cada argumento. Dado que es en el acto de dar y recibir respuestas dónde se hacen evidentes los presupuestos de base que se encuentran presentes en el debate, no basta con analizar asépticamente cada uno de los argumentos de forma aislada. Es en la interacción comunicativa y argumentativa misma donde los presupuestos y concepciones básicas se hacen más fácilmente perceptibles. Sin pretensiones de máxima exhaustividad, a continuación se analizará el debate entre realismo y anti-realismo a través de las óptica de IP y ANM.

\subsection{Las críticas a la Inducción Pesimista}

Las críticas más significativas realizadas a la IP desde el punto de vista de su forma lógica son dos: se le acusa de (i) caer en la denominada The Base Rate Fallacy y, (ii) asumir como punto de partida la tesis que pretende refutar.

Respecto a (i), Lewis $(2001,374)$ señala que la IP interpreta la fiabilidad en términos probabilísticos, pudiéndose dar el caso de la existencia de crecientes positivos verdaderos a lo largo de la Historia de la Ciencia. Teniendo en cuenta que la información acumulada y el descarte de teorías falsas, las probabilidades de dar con la teoría verdadera son cada vez mayores con el paso del tiempo; la inducción histórica, más que prestarse al pesimismo, tendría que ser por ello de calado positivo (Nola 2008). No obstante, tal y como ha apuntado Saatsi (2005, 1094-1098), la variabilidad estadística queda fuera del marco de consideraciones de la IP de Laudan. Tal y como ha sido presentada, la IP no necesita una base estadística sólida para que el argumento sea eficaz; mas bien, basta con encontrar un solo caso en la Historia de la Ciencia donde una teoría científica empíricamente exitosa resultase posteriormente falsa $(\exists \mathrm{t}(\mathrm{St} \wedge \neg \mathrm{Vt}))$ para dar pie a la conclusión del argumento y, por tanto, anular la conexión entre éxito empírico y verdad. 


\section{Sergio Urueña López \\ Los problemas de la inducción pesimista. Verdad y certeza en el debate entre el realismo y el anti-realismo}

Respecto a (ii), Mizrahzi (2013, 3215-3216) sostiene que el hecho de evaluar las teorías actuales como falsas implica presuponer que las actuales son verdaderas, en tanto son estas últimas a la luz de las cuales se juzgan las primeras. Si se sabe que las teorías anteriores fueron falsas, es porque suponemos que las actuales -que son las que sirven como marco evaluativo para Mizrahzi- son efectivamente ciertas. A pesar de que este argumento pueda parecer convincente para el realismo, alguien afín al anti-realismo podría fácilmente replicar que no resulta necesario suponer que las teorías actuales son realmente verdaderas para evaluar las anteriores como falsas. De hecho, podemos suponer que dadas tres teorías científicas $\mathrm{Q}_{\mathrm{T} 1}, \mathrm{R}_{\mathrm{T} 2}$ y $\mathrm{S}_{\mathrm{T} 3}$ con el mismo ámbito de aplicabilidad y situadas en el tiempo $\left(_{\mathrm{T}}\right)$ de tal modo que T3>$\mathrm{T} 2>\mathrm{T} 1$, siempre podría juzgarse a $\mathrm{Q}_{\mathrm{T} 1}$ como falsa a la luz de $\mathrm{R}_{\mathrm{T} 2}$ aun cuando a partir de $\mathrm{S}_{\mathrm{T} 3}$ esta se evidencie también como falsa. Por lo tanto, aun cuando podría darse el supuesto de que se supusiera virtualmente que las teorías actuales son en cierto modo verdaderas, ello no implica aseverar que realmente estas lo son: en tanto no poseemos garantía alguna de que en el futuro sean "superadas" o "refutadas". Además, esta objeción parece asumir que toda teoría puede evaluarse únicamente a la luz de otra, hecho que necesitaría una amplia justificación desde el punto metodológico y epistémico.

Por otro lado, entre las diversas críticas planteadas a la IP que tienen como objeto crítico específico sus premisas, cabria destacar cuatro:

1. Formular que las teorías actuales se distinguen de las pasadas, ya sea (1.a.) mostrando que incluso cuando algunas teorías pasadas fueron exitosas, muchas de ellas no lograron establecer "predicciones novedosas" (Leplin, 1997, cap. 6; Saatsi, 2009, 358), ya (1.b.) señalando las virtudes metodológicas de las teorías maduras frente a las de las refutadas (Hardin y Rosenberg, 1982; Devitt, 1984, 146; Musgrave, 1985, 211; Worrall, 1994; Saatsi, 2009, 358; Park, 2011, 80) y la existencia del crecimiento exponencial de la ciencia (Fahrbach, 2011).

No obstante, Lyons ha señalado que "se pueden ofrecer numerosos ejemplos de predicciones novedosas a partir de teorías que son claramente falsas a las luces del presente" (Lyons, 2003, 898-899; traducción propia). Además, el hecho de que las teorías actuales se distingan de las anteriores no supone para la IP de Laudan un problema mayor: puede que las teorías actuales sean más exitosas y que posean propiedades específicas que las distingan positivamente respecto a las anteriores, pero en cualquier caso ese éxito y esas propiedades no nos aportan garantías sobre su veracidad.

2. Mostrar la existencia de una continuidad a través del cambio teórico que permita explicar el éxito empírico de las teorías pasadas. Esto es, fundamentar que algunas teorías pasadas fueron en algunos aspectos exitosas y, por tanto, parcialmente verdaderas (Worrall, 1989; Psillos, 1994; Nola, 2008). 


$$
\begin{gathered}
\text { Sergio Urueña López } \\
\text { Los problemas de la inducción pesimista. Verdad y certeza en el debate } \\
\text { entre el realismo y el anti-realismo }
\end{gathered}
$$

No obstante, podría replicarse a 2. que mostrar la existencia de cierta continuidad más que asegurar la preservación de verdad meramente explica la preservación del éxito. La cuestión sobre la relación entre el éxito y la verdad seguiría estando pendiente de justificar para los defensores de IP.

3. Defender que los constituyentes teóricos que se consideraron como falsos no poseían en realidad un peso relevante en el éxito de las teorías pasadas (McMullin, 1987; Psillos, 1994; Leplin, 1997).

La réplica más inmediata a esta objeción -pasando por alto lo que una interpretación holista de la confirmación pudiera plantear- es que esta estrategia ex post podría servir para justificar la verdad y el éxito de cualquier teoría, promoviendo una actitud realista demasiado amplia y liberal. En cualquier caso, con IP no se niega (ni se afirma) que existan ciertos elementos que puedan tener un peso más relevante en hacer una teoría más o menos exitosa empíricamente. Lo que se pone en duda es que eso sea un indicador que garantice su verdad.

4. Señalar que la noción de "éxito" manejada en la IP es (de manera intencional) sumamente amplia, permitiéndole basarse en una amplia lista de teorías científicas que hoy en día realmente no consideraríamos exitosas (Carrier, 1991).

A pesar de que esta objeción pueda resultar pertinente, tal y como Laudan señaló ante literam, el criterio de éxito utilizado por él es el mismo que el que mantenían algunos de sus autores realistas coetáneos (Laudan, 1981, 23 y ss.). Por otra parte, y aún cuando pudiera darse el caso de que hoy manejásemos otra noción de éxito diferente a la utilizada por Laudan, la dificultad principal sigue estando presente: el éxito de las teorías (independientemente de como lo interpretemos) no garantiza su verdad y, por lo tanto, su argumento no perdería efectividad. Además, estrechar o ampliar en exceso la noción de éxito podría ser una estrategia contraproducente para el realismo, en tanto puede llevarle a reconocer que muchas de las teorías actuales no cumplen el requisito de éxito fijado.

\subsection{Las críticas al argumento del no-milagro}

En lo que respecta a las críticas realizadas a la forma lógica de ANM, cabe destacar de nuevo dos grupos generales: (i) aquellas enfocadas en señalar que el argumento es circular, y (ii) aquellas centradas en mostrar que este argumento comete la ya conocida The Base Rate Fallacy (véase Howson, 2013).

En lo referente a su forma lógica circular se distinguen a su vez dos críticas: la realizada por Fine (1986) y la realizada por van Fraassen $(1980,21)$. Mientras que Fine argumenta que se trata de un argumento circular en tanto hace uso de la misma lógica que la ciencia a la hora de justificar el conoci- 


$$
\begin{gathered}
\text { Sergio Urueña López } \\
\text { Los problemas de la inducción pesimista. Verdad y certeza en el debate } \\
\text { entre el realismo y el anti-realismo }
\end{gathered}
$$

miento científico (es una IME que justifica las IMEs), van Fraassen señala que la IME es circular porque parte de la presuposición del realismo para afirmarlo, a saber, parte de que toda regularidad precisa de explicación (da por supuesto que bajo las regularidades hay entidades observables) y que la mejor explicación puede considerarse como verdadera.

Las réplicas más inmediatas realizadas a estas dos críticas han pasado por mostrar que esta circularidad no es letal y/o que puede salvarse (véase Musgrave, 1985). Tal es el caso, por ejemplo, de las respuestas ofrecidas por Boyd $(1984,72)$ y Lipton $(1991,164)$, quienes señalaron que la circularidad puede ser relativa a la audiencia y a su nivel de escepticismo respecto a la validez de los procesos inductivos y abductivos (véase la segunda nota al pié del presente trabajo). Además, otra de las réplicas que recientemente se han realizado a este contra-argumento es el interpretar como una IME aquellas posturas que pretenden deslegitimar el uso de IMEs y, de este modo, mostrar que estas posturas podrían aplicarse las mismas objeciones que realizan a ANM. En concreto Mizrahi (2018) lo aplica al caso del Empirismo Constructivo (EC) de van Fraassen:

1. La mejor tesis interpretativa para la actividad científica es EC.

2. Ninguna hipótesis explica tan bien la actividad científica como lo hace EC.

$\therefore$ Por lo tanto, EC es (probablemente) una postura plausible.

El segundo grupo de críticas generales orientadas hacia ANM están encauzadas a determinar que este argumento cae en la ya conocida The Base Rate Fallacy. Magnus y Callender (2004), por ejemplo, interpretan el ANM como un argumento probabilístico. Entendido de tal modo, ANM precisaría basarse en las tasas de éxito de todas las teorías en general y de aquellas que son aproximadamente verdaderas para concluir de manera fehaciente que la proximidad a la verdad incrementa la probabilidad de ser exitosa (algo que resulta imposible de determinar, en tanto no podemos conocer con exhaustividad cuáles son verdaderas). No obstante, diversos autores enfocados en el estudio de las IME sostienen que ANM podría salvar esta objeción si (i) se aplica localmente (y no globalmente - véase Henderson, 2017), así como si (ii) se interpreta ANM como una IME que no concluye que la hipótesis con mayor valor explicativo es de facto la Verdadera (esto es, si comprendemos que afirmar que la mejor explicación del éxito de nuestras teorías es suponer su verdad, no implica afirmar que nuestras teorías sean Verdaderas - véase Diéguez, 1998b).

Respecto a las objeciones orientadas a las premisas o supuestos del ANM, es posible encontrar desde aquellas que afirman que ANM "no puede soportar las tesis realistas" en tanto "no funciona en el plano empírico" (Carrier, 1991, 


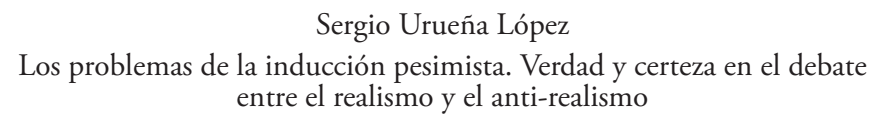

35) hasta aquellas que defienden que "parece no existir una buena razón para preferir el Realismo Científico (en su versión epistémica) sobre otras explicaciones alternativas" (Mizrahi, 2012) -como podría ser el Empirismo Constructivo de van Fraasen $(1980 ; 1989)$. Sin contar, además con aquellas que sostienen que no hay nada que garantice que aquello que consideramos "mejores explicaciones" no sean de hecho un "mal lote" (véase Khalifa, 2010).

En cualquier caso, otro de los puntos focales suele ser la noción de éxito empleada por el realismo en ANM y la supuesta inferencia de verdad que se extrae a partir del mismo. Así, por ejemplo, Wray $(2013,1725-1727)$ señala que la noción de éxito científico no goza de estabilidad diacrónica, por lo que aquello que consideramos "exitoso" sería arbitrario y relativo al periodo específico al que nos encontramos: todas las teorías científicas de cada momento histórico han sido consideradas exitosas dentro del marco histórico del que emergen (al igual que es de esperar que las teorías actuales y futuras sigan siendo consideradas exitosas). Una segunda objeción relacionada con la noción de éxito tiene que ver con la necesidad de vincularla a la noción de verdad (Wray, 2010, 2013). La crítica que afirma que no resulta razonable creer que una teoría es verdadera en base a su éxito empírico se fundamenta ya sea (a) en la posibilidad de construir / dar con una teoría rival T' que posea la misma "fiabilidad instrumental" (Fine, 1986) o "adecuación empírica" (van Fraasen, 1980; 1989) que la inicialmente considerada como más exitosa, y (b) a la falta de garantías existentes entre el éxito empírico y la verdad (Laudan 1981). Se cierra el círculo, entonces, con IP.

\section{Escepticismo, verdad y certeza en el debate entre realismo vs anti-rea- lismo: el caso IP/ANM}

Tras haber analizado en el apartado anterior las diferentes réplicas y contra-argumentos posibles esgrimidos en contra y a favor de IP y ANM, cabría plantearse hasta qué punto el desacuerdo existente entre realistas y anti-realistas está lejos de resolverse y cuáles son los posibles motivos de ello. A la hora de analizar ANM e IP, Dicken (2016) intuye que la falta de entendimiento entre realistas y anti-realistas puede estar basada en el compromiso hacia diferentes presupuestos. Precisamente aquí trataré de defender que uno de esos compromisos y presupuesto de base es el relativo al concepto de verdad que subyace a cada argumento. Ambas posiciones se pronuncian respecto a las posibilidades de verdad de nuestras teorías científicas, pero parecen hacerlo partiendo de marcos conceptuales diferenciados. Mientras que el anti-realismo (al menos con la IP de Laudan) parte del escepticismo para nadar en la amplitud de posibilidades y mostrar nuestra incapacidad a la hora de asegurar que hemos escogido una posición correcta -ello se podría aplicar también al caso de IP de las alternativas no concebidas (Sterpetti y Bertolaso, 2018)-, el realismo (usando ANM) parte del hecho de que podemos conocer de forma 


$$
\begin{gathered}
\text { Sergio Urueña López } \\
\text { Los problemas de la inducción pesimista. Verdad y certeza en el debate } \\
\text { entre el realismo y el anti-realismo }
\end{gathered}
$$

más o menos perfecta la realidad para argumentar a favor de que el conocimiento científico es un conocimiento justificado y razonable (y, por tanto, legitimado para ser interpretado como verdadero).

De manera más concreta, mientras que la persona afín al anti-realismo a través de IP recalca la falta de garantías existentes a la hora de vincular el éxito empírico con la verdad, la realista continúa manteniendo que el éxito es un claro indicador de su verdad. Y el debate se mantiene estancado en estos términos. El anti-realismo sigue advirtiendo que "no es suficiente para el realista socavar la aseveración de que el éxito no es un indicador fiable de la verdad" y que más bien este "debe demostrar que nuestras mejores teorías actuales son probablemente verdaderas" (Wray, 2013, 1719; énfasis original) $y$, por lo tanto, (de)mostrar que no nos encontramos hoy en día en una situación similar a aquellas en las que teorías empíricamente exitosas, resultaron ser posteriormente falsas. Ante ello, quizá el realista podría pensar que la objeción de Laudan (la IP) -y el reto de Wray- es insalvable y que por tanto tratar de seguir manteniendo su posición mediante ANM sería un acto destinado al fracaso (en tanto su poder reside en tomar el éxito como un indicador de la verdad de nuestras teorías). No obstante, cabe preguntarse hasta que punto ello es así. La IP de Laudan parece ser un argumento claramente válido y pertinente, pero ¿en qué medida afecta realmente al realismo en general y a ANM en particular?

En mi opinión, a la hora de tratar de afrontar esta pregunta, merecería la pena tanto (i) analizar la forma lógica del argumento, como (ii) examinar el concepto de verdad que subyace a IP. Para ello, puede ser una tarea especialmente útil el recordar, por un lado, que el objetivo central de IP es mostrar la ausencia de garantías de la relación éxito empírico-verdad (sección 3) y que, para superar el argumento, los propios anti-realistas establecen como condición necesaria el (de)mostrar que las teorías actuales son de hecho verdaderas (y que no logran simplemente "salvar el fenómeno") (véase Wray, 2013).

Tal y como se mencionó en la segunda sección del presente trabajo, la inducción pesimista posee la siguiente forma lógica:

$$
\frac{\exists \mathrm{t}(\mathrm{St} \wedge \neg \mathrm{Vt})}{\therefore \neg \forall \mathrm{t}(\mathrm{St} \rightarrow \mathrm{Vt})}
$$

Una forma lógica que comparte ciertas similitudes con algunos argumentos escépticos ampliamente conocidos en el ámbito filosófico. Piénsese, por ejemplo, en el siguiente: "dado que existen percepciones $(\mathrm{t})$ que se nos presentan como vívidas $(\mathrm{S})$ pero que han resultado ser falsas $(\neg \mathrm{V})$, no toda percepción que se nos presente como vívida puede tomarse como fiable". De ahí pasaría a extraerse que no tenemos garantías de que las percepciones que se nos presentan como vívidas sean de hecho verdaderas y que, por tanto, no 


$$
\begin{aligned}
& \text { Sergio Urueña López } \\
& \text { Los problemas de la inducción pesimista. Verdad y certeza en el debate } \\
& \text { entre el realismo y el anti-realismo }
\end{aligned}
$$

podemos tomar la percepción como una fuente certera de conocimiento. $\mathrm{La}$ sombra del escepricismo se extiende: ¿Cómo podemos estar seguros de que aquello que percivimos vívidamente no es más que una ilusión?

La IP comparte con este argumento escéptico, además de la fórmula lógica, la fijación por la certeza: lo que ambos pretenden señalar es que no tenemos una prueba definitiva acerca de la conexión éxito empírico/vividez perceptiva y la verdad. Y ciertamente es así, no poseemos tal prueba; pero, ¿es posible tenerla? ¿necesitamos realmente esa prueba a la hora de defender el realismo como una postura filosófica razonable a la hora de interpretar nuestras teorías científicas? Convendría recordar las siguientes palabras de Popper a la hora de responder la pregunta acerca de la posibilidad de la prueba:

El realismo no es demostrable ni tampoco refutable. Al igual que todo lo que caiga fuera del campo de la Lógica y de la Aritmética finita, el realismo no es demostrable; mientras que las teorías científicas empíricas son refutables, el realismo ni siquiera lo es. (...) Con todo, se puede argumentar, y el peso de los argumentos está abrumadoramente a su favor. (Popper, 1989, 39; traducción propia).

Ciertamente, no existe tal demostración que ofrezca a los anti-realistas la certeza requerida sobre las tesis realistas, pero ello no implica que el realismo haya sido refutado por la IP ni que esta haya dejado de ser una tesis plausible o razonable (Diéguez, 1998b; 2007). El anti-realismo está solicitando al realismo a la hora de evaluar su razonabilidad una tarea que a priori sabe que es imposible de asumir (la misma que los críticos de la inducción demandaban a la lógica inductiva). No podemos demostrar de manera necesaria y suficiente que todo lo que estamos viviendo no es una mera ensoñación, que no somos cerebros metidos en una cubeta o que nuestras teorías científicas son verdaderas. La dificultad de superar la inducción pesimista es sin duda la propia que a nivel teórico poseen las tesis profundamente escépticas: dentro de su estricto marco de operatividad, no son superables. Por ello, la única salida es poner en cuestión ese mismo marco bajo el que operan.

En sentido estricto, ante la ausencia de posibilidad de brindar una prueba que le sirva a la anti-realista como justificación necesaria y suficiente -esto es, la propia que ofrecen los razonamientos deductivos ${ }^{2}-$, no podemos garantizar que el progreso de la ciencia haya alcanzado cotas gradientes de "verdadera verosimilitud" (o de Verdad, en mayúscula):

\footnotetext{
${ }^{2} \mathrm{El}$ anclaje del concepto de verdad a la certeza puede estar funcionando a nivel meta-argumentativo. Aunque aquí no cabe desarrollar esta hipótesis, resultaría interesante estudiar la posibilidad de que el rechazo al ANM (entendido como IME) por parte de los defensores de IP, se deba en buena parte a que este no presenta una forma lógica deductiva y, por tanto, no sea garante lógico de aquello que proclama. Dicho con otras palabras, la crítica al ANM
} 


$$
\begin{gathered}
\text { Sergio Urueña López } \\
\text { Los problemas de la inducción pesimista. Verdad y certeza en el debate } \\
\text { entre el realismo y el anti-realismo }
\end{gathered}
$$

Niiniluto ha mostrado que (...) la 'verosimilitud estimada' es una medida de cómo de cercano (el contenido de) una teoría se corresponde a lo que consideramos los mejores sistemas conceptuales que hasta ahora hemos sido capaces de encontrar (...). Pero debido a que la verosimilitud estimada y la genuina verosimilitud no están necesariamente relacionadas (...) es una cuestión abierta si -como Niiniluoto dice- el éxito continuo de las teorías altamente confirmadas puede ser explicado por la hipótesis de que, de hecho, están cerca de la verdad al menos en los aspectos relevantes. Si no me equivoco, esta observación delata una confusión entre la 'verdadera verosimilitud' (a la que no tenemos acceso epistémico) y la 'verosimilitud estimada' (que es accesible, pero no-epistémica). (Laudan, 1981, 31, n.10; traducción propia).

La distinción entre "verdadera verosimilitud" y la "verosimilitud estimada" puede ser de gran ayuda a la hora de seguir ilustrando que el concepto de verdad manejado en IP tiene su base en la certeza. Laudan reconoce que la verdadera verosimilitud no es accesible (y por tanto no "mostrable"), pero no acepta que puedan existir otras formas epistémicas de verdad que resulten fértiles y explicativas a la hora de interpretar la práctica científica (al margen de los problemas que el concepto de "verosimilitud estimada" pueda presentar). Cuando Laudan afirma por tanto en A Confutation of Convergent Realism (1981) que el éxito empírico no implica la verdad de la ciencia, esto es, que $\neg \forall \mathrm{t}(\mathrm{St} \rightarrow \mathrm{Vt})$, está tomando la verdadera verosimilitud, la Verdad en mayúsculas, como marco de referencia general desde el que evaluar al realismo. Pero este concepto de Verdad al no ser epistémicamente accesible y/o al estar ligado a la certeza, es tan sumamente restrictivo que resultaría inoperativo a la hora de interpretar y proponer criterios de validación de cualquier tipo de conocimiento más allá de los justificados mediante tautologías, métodos deductivos y verdades triviales. Dicho de otro modo, Laudan está interpretando el realismo desde un punto de vista puramente semántico y bajo un nivel de radicalidad bastante alto (y, por tanto, sólo aplicable a esos mismos realismos más "ingenuos").

Cabe no obstante asumir la posibilidad teórica de que el realismo en general y el ANM en particular operen bajo otro concepto de verdad no tan restrictivo, mucho más modesto y alejado de las concepciones semánticas más radicales. En este sentido, la IP puede ser fácilmente asumida por el realismo,

puede deberse no tanto al argumento mismo, sino a su forma: es una crítica general a los argumentos de corte abductivos e inductivos (véase Howson 2000). De ahí que la disputa entre Fine (1986) y Boyd (1984: 72-72) o Lipton (1991: 164) a la que se hacía referencia en la sección 3, pueda comprenderse en estos términos: la intuición de que ANM es sólo válido para el realismo podría deberse a que estos reconocen la validez de argumentos que van más allá de la deducción a la hora de defender una hipótesis. 


$$
\begin{gathered}
\text { Sergio Urueña López } \\
\text { Los problemas de la inducción pesimista. Verdad y certeza en el debate } \\
\text { entre el realismo y el anti-realismo }
\end{gathered}
$$

siempre que no se asuma un concepto de verdad epistémicamente anclado en la certeza y se reconozca la falibilidad, la dimensión pragmática y el carácter siempre perfectible de nuestro conocimiento:

No hay garantía, por supuesto, de que este proceso de aprendizaje de las experiencias pasadas nos conduzca de teorías falsas a otras más verdaderas. Sin embargo, si los científicos pueden aprender de la experiencia pasada, están en una mejor posición para abandonar afirmaciones teóricas falsas a favor de las nuevas que están mejor soportadas por la evidencia. Por lo tanto, estas afirmaciones es más probable que sean verdaderas que aquellas que ya han sido abandonadas. (Psillos, 1999, 99; traducción propia).

Dicho de otro modo, el realismo puede perfectamente aceptar que: “ $\neg \forall \mathrm{t}$ $(\mathrm{St} \rightarrow \mathrm{Vt})$ ", sin tener que renunciar por ello a sostener que "St $\rightarrow \mathrm{Vt}$ " o que " $\exists \mathrm{t}(\mathrm{St} \rightarrow \mathrm{Vt})$ " entendiendo tanto la implicación como la verdad en un sentido deflacionario: esto es, entendiendo la conexión éxito-verdad no en términos de necesidad, sino comprendiendo el éxito como uno de los indicadores de la verdad, y esta última como una convención basada en criterios epistémica y metodológicamente robustos. Dicho de otro modo, es posible entender la conexión éxito empírico-verdad de una manera tentativa y como un criterio para comprender o interpretar las teorías científicas actuales ${ }^{3}$.

En este sentido, conviene señalar que el realismo (y ANM) no es una posición sobre la Verdad de las teorías científicas, sino más bien una hipótesis (interpretativa) sobre la razonabilidad de creer en nuestras teorías científicas actuales como verdaderas en tanto suficientemente justificadas desde un punto de vista epistémico. Que las teorías científicas logran salvar el fenómeno es ciertamente un dato empírico. Pero el realismo científico va más allá de ese dato para ofrecer una hipótesis interpretativa / explicativa del mismo: invita a considerar, y a modo de tentativa, las teorías científicas como verdaderas. Una cosa es describir qué hace la ciencia, y otra es tratar de explicar por qué ello es así; $y$, a la hora de proponer una explicación del poder y valor predictivo, explicativo, heurístico, instrumental, etc. de nuestras teorías científicas, el ANM se erige como el argumento más plausible frente a la IP de Laudan y, por tanto, el realismo se erige como una posición sensata a la hora de valorar y comprender la praxis científica.

\footnotetext{
${ }^{3}$ Este carácter tentativo, por otra parte, es lo que permitía "superar", para Reichenbach, el escepticismo humeano: "puede darse una salida al escepticismo de Hume cuando el conocimiento es concebido no como un sistema de proposiciones que tienen un valor de verdad determinable o un valor de probabilidad, sino como un sistema de tentativas" (Reichenbach, 1951, 49; traducción propia).
} 


$$
\begin{gathered}
\text { Sergio Urueña López } \\
\text { Los problemas de la inducción pesimista. Verdad y certeza en el debate } \\
\text { entre el realismo y el anti-realismo }
\end{gathered}
$$

\section{Conclusiones}

A través del presente trabajo se han llegado a las siguientes conclusiones:

1. Tanto "ANM" como "IP" denotan una variedad de argumentos, siendo esta diversidad especialmente significativa en el caso de IP (sección 2).

2. Los contra-argumentos ofrecidos a la IP de Laudan no parecen ser efectivos (sección 3).

3. La inefectividad de los argumentos contra la IP de Laudan se debe a que el argumento opera con un concepto de verdad cuya categoría epistémica central es la certeza (es verdadero todo aquello que pueda creerse y/o justificarse con certeza) (sección 4).

4. Esta rigidez epistémica ligada a la certeza podría estar funcionando no sólo en las bases del argumento sino también en el marco metaargumentativo: al ser ANM un argumento típicamente abductivo, este no ofrece garantías últimas de sus conclusiones; mientras IP, al ser un argumento deductivo, asegura la verdad de sus conclusiones dada la verdad de las premisas (sección 4).

5. Si bien la IP de Laudan es un buen argumento, el concepto de verdad bajo el que este opera resulta inoperativo para comprender y justificar el conocimiento generado por la práctica científica (en tanto conocimiento tentativo y falible) (sección 4).

6. El realismo podría erigirse como una hipótesis interpretativa adecuada frente a IP siempre que no ancle su concepto de verdad a la certeza. Así entendido, ANM sigue siendo un argumento válido: resulta razonable pensar en las teorías científicas actuales como verdaderas siempre que se comprenda esa "verdad" en un sentido hipotético y sin que ello signifique abandonar una perspectiva crítica de la práctica científica (sección 4).

\section{Agradecimientos}

Agradezco los comentarios y sugerencias ofrecidos por los/as asistentes a las I Jornadas Novatores "Enfoques contemporáneos del Realismo Científico" (Salamanca, 11-12 de abril de 2018) donde se presentó una versión preliminar del presente trabajo. El autor disfruta de un contrato predoctoral para la Formación de Personal Investigador (BES-2016-079192, MINECO/ FEDER) enmarcada en el proyecto "Representación y Anticipación: modelización interventiva RRI en las ciencias y técnicas emergentes" (FFI201569792-R, MINECO/FEDER). 


$$
\begin{aligned}
& \text { Sergio Urueña López } \\
& \text { Los problemas de la inducción pesimista. Verdad y certeza en el debate } \\
& \text { entre el realismo y el anti-realismo }
\end{aligned}
$$

\section{Referencias bibliográficas}

Boyd, Richard (1984). The current status of scientific realism. En Jarrett Leplin (Ed.), Scientific Realism (pp. 41-82). Berkeley: University of California Press.

Brown, James R. (1982). The Miracle of Science. Philosophical Quarterly, 32(128), 232-244.

Carman, Christián C. (2006). El realismo científico y los milagros inesperados. Ludus Vitalis, 14(26), 93-101.

Carman, Christián C. (2016). Realismo científico. En Claudia E. Vanney, Ignacio Silva y Juan F. Franck (Eds.), Diccionario Interdisciplinar Austral. Obtenido de http://dia.austral.edu.ar/Realismo_científico.

Carrier, Martin (1991). What is wrong with the miracle argument? Studies in History and Philosophy of Science, 22(1), 23-36.

Chakravartty, Anjan (2017). Scientific Realism. En Edward N. Zalta (Ed.), The Standford Encyclopedia of Philosophy. Obtenido de https://plato. stanford.edu/archives/sum2017/entries/scientific-realism/.

Dellsén, Finnur (2016). Explanatory Rivals and the Ultimate Argument. Theoria, 82(3), 217-237.

Devitt, Michael (1984). Realism and Truth. Oxford: Blackwell.

Dicken, Paul (2016). A Critical Introduction to Scientific Realism. London: Bloomsbury Academic.

Diéguez, Antonio (1998a). Realismo científico. Una introducción al debate actual en la filosofía de la ciencia. Málaga: Universidad de Málaga.

Diéguez, Antonio (1998b). Los compromisos del realismo científico. Contrastes. Revista Interdisciplinar de Filosofía, 3, 145-173.

Diéguez, Antonio (2007). Why Does Laudan's Confutation of Convergent Realism Fail? Journal for General Philosophy of Science, 37, 393-403.

Fahrbach, Ludwig (2011). How the Growth of Science Ends Theory Change. Synthese, 180(2), 139-155.

Fine, Arthur (1984). The Natural Ontological Attitude. En Jarret Leplin (Ed.), Scientific Realism (pp. 61-77). Berkeley: University of California Press.

Fine, Arthur (1986). Unnatural Attitudes: Realist and Instrumentalist Attachments to Science. Mind, 95(378), 149-179.

González, Wenceslao (1993). El realismo y sus variedades: el debate actual sobre las bases filosóficas de la Ciencia. En Antonio Carreras (Ed.), Conocimiento, Ciencia y Realidad (pp. 11-58). Zaragoza: SIUZ. 


$$
\begin{gathered}
\text { Sergio Urueña López } \\
\text { Los problemas de la inducción pesimista. Verdad y certeza en el debate } \\
\text { entre el realismo y el anti-realismo }
\end{gathered}
$$

Hardin, Clyde L. y Rosenberg, Alexander (1982). In Defence of Convergent Realism. Philosophy of Science, 49(4), 604-615.

Henderson, Leah (2017). The no miracles argument and the base rate fallacy. Synthese, 194(4), 1295-1302.

Howson, Colin (2000). Hume's problem: Induction and the justification of belief. Oxford: Oxford University Press.

Howson, Colin (2013). Exhuming the No-Miracles Argument. Analysis, 73(2), 205-211.

Khalifa, Kareem (2010). Default Privilege and Bad Lots: Underconsideration and Explanatory Inference. International Studies in the Philosophy of Science, 24(1), 91-105.

Kitcher, Philip (1993). The Advancement of Science. Oxford: Oxford University Press.

Lange, Marc (2002). Baseball, pessimistic inductions and the turnover fallacy. Analysis, 62(276), 281-285.

Laudan, Larry (1977). Progress and Its Problems: Towards a Theory of Scientific Growth. California: University of California Press.

Laudan, Larry (1981). A Confutation of Convergent Realism. Philosophy of Science, 48(1), 19-49.

Leplin, Jarrett (1997). A Novel Defense of Scientific Realism. New York: Oxford University Press.

Levin, Michael (1984). What kind of explanation is Truth? En Jarret Leplin (Ed.), Scientific Realism (pp. 124-139). Berkeley: University of California Press.

Lewis, Peter J. (2001). Why The Pessimistic Induction Is A Fallacy. Synthese, 129(3), 371-380.

Lipton, Peter (1991). Inference to the best explanation. London: Routledge.

Lyons, Timothy D. (2002). Scientific Realism and the Pessimistic Meta-Modus Tollens. En Steve Clarke y Timothy D. Lyons (Eds.), Recent Themes in the Philosophy of Science: Scientific Realism and Commonsense (pp. 63-90). Dordrecht: Kluwer.

Lyons, Timothy D. (2003). Explaining the Success of a Scientific Theory, Philosophy of Science, 70, 891-901. 


$$
\begin{gathered}
\text { Sergio Urueña López } \\
\text { Los problemas de la inducción pesimista. Verdad y certeza en el debate } \\
\text { entre el realismo y el anti-realismo }
\end{gathered}
$$

Lyons, Timothy D. y Clarke, Steve (2002). Scientific Realism and Commonsense. En Steve Clarke y Timothy D. Lyons (Eds.), Recent Themes in the Philosophy of Science: Scientific Realism and Commonsense (pp. ix-xxiii). Dordrecht: Kluwer.

Magnus, P.D. y Callender, Craig (2004). Realist Ennui and the Base Rate Fallacy. Philosophy of Science, 71(3), 320-338.

Maxwell, Grover (1962). The Ontological Status of Theoretical Entities. En Herbert Feilg y Grover Maxwell (Eds.), Scientific Explanation, Space, and Time (pp. 3-27). Minnesota: University of Minnesota Press.

McMullin, Ernan (1987). Explanatory Success and the Truth of Theory. En Nicholas Rescher (Ed.), Scientific Inquiry in Philosophical Perspective (pp. 51-73). Lanham: University Press of America.

Mizrahi, Moti (2012). Why the ultimate argument for scientific realism ultimately fails. Studies in History and Philosophy of Science, 43, 132-138.

Mizrahi, Moti (2013). The Pessimistic Induction: A Bad Argument Gone Too Far. Synthese, 190(15), 3209-3226.

Mizrahi, Moti (2018). The "Positive Argument" for Constructive Empiricism and Inference to the Best Explanation, Journal for General Philosophy of Science, 1-6.

Musgrave, Alan (1985). Realism vs. Constructive Empiricism. En Paul M. Churchland y Clifford A. Hooker (Eds.), Images of Science: Essays on Realism and Empiricism (pp. 197-221). Chicago: The University of Chicago Press.

Musgrave, Alan (1988). The Ultimate Argument for Scientific Realism. En Robert Nola (Ed.), Relativism and Realism in Science (pp. 229-252). Dordrecht: Kluwer.

Nola, Robert (2008). The optimistic meta-induction and ontological continuity: the case of the electron. En Léna Soler, Howard Sankey y Paul Hoyningen-Huene (Eds.), Rethinking scientific change and theory comparison: Stabilities, ruptures, incommensurabilities (pp. 159-202). Dordrecht: Springer.

Olmos, Paula (pendiente de publicación). Un enfoque argumentativo sobre la abducción y sobre la ponderación de hipótesis explicativas. Theoria.

Park, Seungbae (2011). A Confutation of the Pessimistic Induction. Journal for General Philosophy of Science, 42(1), 75-84.

Peirce, Charles S. (1958). Collected Papers. Vol. 5. Cambridge: Harvard University Press. 


$$
\begin{gathered}
\text { Sergio Urueña López } \\
\text { Los problemas de la inducción pesimista. Verdad y certeza en el debate } \\
\text { entre el realismo y el anti-realismo }
\end{gathered}
$$

Poincaré, Henry ([1905] 2001). Science and Hypothesis. En Henry Poincaré, The Value of Science: Essential Writings of Henri Poincaré (pp. 1-178). Nueva York: Dover.

Popper, Karl (1989). Objective knowledge: An evolutionary approach. Oxford: Oxford University Press.

Psillos, Stathis (1994). A Philosophical Study of the Transition from the Caloric Theory of Heat to Thermodynamics: Resisting the Pessimistic Meta-Induction. Studies in History and Philosophy of Science, 32, 691-710.

Psillos, Stathis (1996). Scientific Realism and the "Pessimistic Induction". Philosophy of Science, 63, S306-S314.

Psillos, Stathis (1999). Scientific Realism: How Science Tracks Truth. Nueva York: Routledge.

Psillos, Stathis (2009). Thinking About the Ultimate Argument for Realism. En Stathis Psillos (Ed.), Knowing the Structure of Nature (pp. 48-68). Londres: Palgrave Macmillan.

Putnam, Hilary (1975). Mathematics, Matter and Method. Vol. 1 of Philosophical Papers. Cambridge: Cambridge University Press.

Putnam, Hilary (1976). What is Realism? Proceedings of the Aristotelian Society, 76(1), 177-194.

Reichenbach, Hans (1951). Bertrand Rusell's Logic. En Paul Arthur Schilpp (Ed.), The Philosophy of Bertrand Russell (pp. 23-54). Nueva York: Tudor.

Saatsi, Juha (2005). On the Pessimistic Induction and Two Fallacies, Philosophy of Science, 72(5), 1088-1098.

Saatsi, Juha (2009). Grasping at Realist Straws. Review Symposium, Metascience, 18: 355-362.

Saatsi, Juha, Psillos, Stathis, Grønfeldt, Rasmus y Stanford, Kyle (2009). Grasping at Realists Straws. Metascience, 18, 355-390.

Smart, John Jamieson C. (1963). Philosophy and scientific realism. London: Routledge.

Stanford, P. Kyle (2001). Refusing the Devil's Bargain: What Kind of Underdetermination Should We take Seriously? Philosophy of Science, 68(Proceedings), S1-S12.

Stanford, P. Kyle (2006). Exceeding our grasp: Science, history, and the problem of unconceived alternatives. Oxford: Oxford University Press. 
Sterpetti, Fabio y Bertolaso, Marta (2018). The Pursuit of Knowledge and the Problem of Unconceived Alternatives. Topoi, 1-12.

van Fraassen, Bas C. (1980). The Scientific Image. Oxford: Oxford University Press.

van Fraassen, Bas C. (1989). Laws and Symmetry. Oxford: Clarendon Press.

Worrall, John (1989). Structural Realism: The best of both worlds. Dialectica, 43(1-2), 99-124.

Worrall, John (1994). How to Remain (Reasonably) Optimistic: Scientific Realism and the 'Luminiferous Ether'. PSA, 1, 334-342.

Worrall, John (2005). Miracles, Pessimism and Scientific Realism. Obtenido de https://philarchive.org/archive/WORMPAv1.

Wray, K. Brad (2010). Selection and predictive success. Erkenntnis, 72(3), 365-377.

Wray, K. Brad (2013). Success and truth in the realism/anti-realism debate. Synthese, 190(9), 1719-1729.

Wray, K. Brad (2015). Pessimistic Inductions: Four Varieties. International Studies in the Philosophy of Science, 1(29), 67-73. 